\title{
Research
}

\section{The Prehospital Ambulance Stroke Test vs. the Cincinnati Prehospital Stroke Scale: a diagnostic accuracy study}

Somayeh Karimi MD, is Emergency Medicine Resident1,2; Hassan Motamed MD, is Associate Professor of Emergency Medicine; Ehsan Aliniagerdroudbari MD, is a general practitioner ${ }^{4}$; Sepideh Babaniamansour MD, is a general practitioner ${ }^{5}$; Arman Jami MD, is a general practitioner ${ }^{4}$; Alireza Baratloo MD, is Associate Professor of Emergency Medicine $e^{1,2}$

\author{
Affiliations: \\ 1Prehospital and Hospital Emergency Research Center, Tehran University of Medical Sciences, Tehran, Iran \\ ${ }^{2}$ Department of Emergency Medicine, Sina Hospital, Tehran University of Medical Sciences, Tehran, Iran \\ ${ }^{3}$ Department of Emergency Medicine, Ahvaz Jundishapur University of Medical Sciences, Ahvaz, Iran \\ ${ }^{4}$ School of Medicine, Shahid Beheshti University of Medical Sciences, Tehran, Iran \\ ${ }^{5}$ School of Medicine, Islamic Azad University of Medical Sciences, Tehran, Iran
}

https://doi.org/10.33151/ajp.17.784

\section{Abstract}

\section{Introduction}

Immediate diagnosis of stroke is crucial in reducing its morbidity and mortality rate. There are various pre-hospital assessment tools, such as the Prehospital Ambulance Stroke Test (PreHAST) and the Cincinnati Prehospital Stroke Scale (CPSS) used to identify stroke early in the chain of care. The aim of this study is to compare the accuracy of PreHAST with CPSS in diagnosing stroke.

\section{Methods}

In this diagnostic accuracy study patients with suspicion of stroke were included in this study. In CPSS, the criterion used to indicate stroke are facial droop, speech and arm drift. In PreHAST it is eye position, visual field, facial palsy, right and left arms paresis, right and left legs paresis, sensory and speech. After data collection, sensitivity and specificity were calculated using standard formulae. Different cut-off points for the best diagnostic accuracy were examined in both CPSS and PreHAST.

\section{Results}

In this study, 883 patients were investigated. The results demonstrated that in CPSS, the highest specificity and sensitivity was for facial droop (84.9\%) and arm drift (82.7\%); and in PreHAST it was eye position (99.6\%) and facial palsy (49.2\%). The best predictor of stroke in CPSS with highest sensitivity (78.5\%) and specificity (66\%) was a cut-off point of 1.5 (AUC: 0.744$)$ ( $p<0.01$ (. In PreHAST, the highest sensitivity $(68.4 \%)$ was a cut-off point of 2.5 and the highest specificity $(90.2 \%)$ was a cut-off point of 5.5 (AUC: 0.775 ) $(p<0.01)$.

\section{Conclusion}

Both PreHAST and CPSS are useful screening tools in the pre-hospital diagnosis of stroke. In addition to high sensitivity, these tests provide a grading system in which higher cut-off points lead to higher specificity.

\section{Keywords:}

stroke; emergency medical services; triage; diagnosis; symptom assessment

Corresponding Author: Alireza Baratloo, alirezabaratloo@yahoo.com 


\section{Introduction}

Stroke is one of the most common causes of disabilityadjusted life years (DALYs) and the third highest cause of death worldwide with a burden of 5.7 million people (1-3). On average, one person dies of stroke every 3 minutes in the United States and $50 \%$ of these deaths take place in the pre-hospital setting (4-6). Brain cells are highly vulnerable to ischaemia, thrombosis of a large vessel can destroy 1.9 million neurons per minute and has a high morbidity and mortality rate - hence the phrase, 'time is brain' (7-11). 2018 American Heart Association and American Stroke Association guidelines state the best way to manage patients with acute ischaemic stroke is immediate diagnosis with high reliability, and a timely start of thrombolytic medications or mechanical clot retrieval (12-15). Both therapeutic methods are time sensitive, therefore stroke diagnosis and determination of its severity in the pre-hospital setting is of paramount importance in order to obtain best results (16-18). Unfortunately, only half of patients with stroke call emergency medical services (EMS) for primary triage and only 15-60 of patients with stroke symptoms are referred to hospital in the first 3 hours (19-21). On the other hand, a study in 2017 estimated the rate of stroke under-diagnosis from $2 \%$ to $26 \%$ and the rate of stroke over-diagnosis from $30 \%$ to $43 \%$ in the emergency department (ED) setting (22). Given the impossibility of performing para-clinical diagnostic tests at pre-hospital stage in most countries, diagnosis and decisionmaking must be done based on clinical presentation. Some studies have investigated the best pre-hospital diagnostic tests based on findings of physical examination with high precision and high sensitivity in the early triage of stroke patients (23). The Cincinnati Prehospital Stroke Scale (CPSS) is one of the most commonly used pre-hospital tests used to assess symptoms based on the simplification of the National Institute of Health Stroke Scale (NIHSS), in which the presence of at least one of the three signs of facial palsy, unilateral arm weakness or speech impairment is considered as positive (24-26). The Prehospital Ambulance Stroke Test (PreHAST) was introduced in 2017 and consists of eight signs based on the NIHSS and a deficit in any sign (score 1 to 19) is defined as positive (16). In a systematic review in 2014 , sensitivity and specificity of CPSS was reported at $79-95 \%$ and $24-56 \%$, respectively; while the sensitivity and specificity of PreHAST (score of equal or more than 1 point) in stroke diagnosis was estimated at $100 \%$ and $40 \%$, respectively $(16,27)$. As PreHAST has not yet been compared to other scales in a similar population, we aimed to compare the accuracy of PreHAST to CPSS in terms of diagnosis of patients referred to an ED by EMS with a suspicion of stroke.

\section{Methods}

\section{Study design}

This diagnostic accuracy study was a multicentre survey conducted in the ED of four teaching hospitals in Iran during
2018 and 2019. The implementation of the project was approved by the Ethics Committee of Tehran University of Medical Sciences (Ethic Code: IR.TUMS.MEDICINE. REC.1398,166). The patients joined the study after taking informed consent and the study did not impose any additional costs on patients or on the health system.

\section{Participants}

Patients more than 18 years of age who underwent a brain magnetic resonance imaging (MRI) with suspicion of stroke in the 12-24 hours after arriving to the ED. Information defect or inconsistencies in the recorded findings were considered as exclusion criteria. According Andsberg et al with assumption of sensitivity at $100 \%$ and specificity at $44 \%$, accuracy at $95 \%$ and error at 5\%, sample size was calculated at at least 996 people. Sampling was carried out retrospectively in sequential manner (16).

\section{Test methods}

Evaluation of stroke was based on PreHAST and CPSS. In CPSS criterion, three signs including facial droop, speech and arm drift were assessed to indicate a stroke, with the presence of one of the three signs considered as positive. PreHAST consists of eight signs: eye position, visual field, facial palsy, right and left arms paresis, right and left legs paresis, sensory and speech. The test scores stroke severity from 0 to 19 points and a score equal or more than 1 point is considered as positive.

All data were collected in a checklist of three sections based on the patient's file records. The first section was about basic information and demographic data including age, gender, past medical history (ischaemic heart disease, seizure, diabetes mellitus, hypertension, hyperlipidemia, stroke and coagulopathy), smoking habits, blood sugar level and the duration from symptom onset to arrival at the ED. Blood sugar was measured by glucometer (Accuchek, Roche, Germany). The second section included physical examination results associated with the eight items of PreHAST and three items of CPSS. The third section was devoted to the final diagnosis based on brain MRI reports confirmed by two radiologists.

\section{Analysis}

All data was added to SPSS version 24 (SPSS Inc., Chicago, IL, USA) and analysed. Continuous variables were described using mean \pm standard deviation and categorial variables were described using frequency and percentage. In order to evaluate frequency among the categorial variables chi-square test and Fisher's exact test were used, one-way ANOVA test and independent t-test were applied to analyse the relationship between categorial and continuous variables. A p-value less than 0.05 was considered as statistically significant. Collected data from PreHAST and CPSS were compared with the final diagnosis after performing Bonferroni correction. Sensitivity, 
specificity, accuracy, positive predictive value (PPV) and negative predictive value (NPV) for three CPSS items and eight PreHAST items were measured separately using statistical tests. In order to examine the strengths of the test and determine the best cut-off point ROC curve and the area under the curve (AUC) were used.

\section{Results}

\section{Participants}

Documents of 1000 patients were investigated, 117 were excluded. Finally, 883 patients with an average age of 67.20 \pm 13.66 years with suspicion of stroke entered the study, 505 $(57.2 \%)$ were men. The final diagnosis was stroke in 618 of the cases (70\%). Basic and demographic information of studied patients is summarised in Table 1.

Table 1. Baseline characteristics of studied patients and their relationship with final diagnosis

\begin{tabular}{|l|c|c|}
\hline \multicolumn{1}{|c|}{ Variable } & $\begin{array}{c}\text { Frequency } \\
\text { (percent) }\end{array}$ & p-value* \\
\cline { 1 - 2 } Gender & $378(42.8 \%)$ & \multirow{2}{*}{0.07} \\
\hline Female & $505(57.2 \%)$ & \\
\hline Male & $134(15.2 \%)$ & 0.75 \\
\hline Past medical history & \multicolumn{3}{|l}{} \\
\hline Smoker & $546(61.6 \%)$ & 0.11 \\
\hline Hypertension & $293(33.2 \%)$ & 0.13 \\
\hline Ischaemic heart disease & $293(33.2 \%)$ & 0.21 \\
\hline Diabetes mellitus & $79(8.9 \%)$ & $<0.01$ \\
\hline Coagulopathy & $174(19.7 \%)$ & 0.40 \\
\hline Stroke & $176(19.9 \%)$ & 0.27 \\
\hline Hyperlipidemia & $30(3.4 \%)$ & 0.06 \\
\hline Seizure &
\end{tabular}

${ }^{*} p$-value refers to the relationship between each variable and final diagnosis of stroke

Previously, only a medical history of coagulopathy had a significant relationship with stroke $(p<0.01)$ (Table 1). Results also showed the incidence of stroke increased significantly with increasing age $(p<0.01)$ and mean level of blood sugar was significantly higher in non-stroke patients $(p=0.04)$. Comparing the duration from symptom onset to arrival at the ED with a stroke diagnosis revealed that patients with stroke diagnosis were significantly referred to the hospital in a shorter time $(p<0.01)$ (Table 2).

\section{Test results}

The chi-square test showed that abnormal cases in each of the three items in CPSS and in each of the eight items in PreHAST were significantly higher in patients with a final diagnosis of stroke $(p<0.05)$ (Table 3). However, after performing Bonferroni correction, arm drift and visual field had no significant difference with the final diagnosis of stroke, but the difference of other items was still statistically significant.

The study also showed that the highest specificity and sensitivity in CPSS was for facial droop (84.9\%) and arm drift (82.7\%); and in PreHAST, eye position (99.6\%) and facial palsy $(49.2 \%)$ (Table 4).

Table 4. Sensitivity and specificity of CPSS and PreHAST items

\begin{tabular}{|c|c|c|c|c|}
\hline \multicolumn{3}{|c|}{ Test } & Sensitivity & Specificity \\
\hline \multirow{3}{*}{ CPSS } & \multicolumn{2}{|c|}{ Facial droop } & $49 \%$ & $84.9 \%$ \\
\hline & \multicolumn{2}{|l|}{ Arm drift } & $82.7 \%$ & $23.4 \%$ \\
\hline & \multicolumn{2}{|l|}{ Speech } & $77.5 \%$ & $60.8 \%$ \\
\hline \multirow{16}{*}{ PreHAST } & \multicolumn{2}{|c|}{ Commands } & $16.7 \%$ & $97 \%$ \\
\hline & \multicolumn{2}{|c|}{ Eye position } & $16.2 \%$ & $99.6 \%$ \\
\hline & \multicolumn{2}{|l|}{ Visual field } & $5.3 \%$ & $98.1 \%$ \\
\hline & \multicolumn{2}{|c|}{ Facial palsy } & $49.2 \%$ & $76.6 \%$ \\
\hline & \multirow{2}{*}{$\begin{array}{l}\text { Right arm } \\
\text { paresis }\end{array}$} & Score 1 & $17.6 \%$ & \multirow{2}{*}{$89.8 \%$} \\
\hline & & Score 2 & $17.6 \%$ & \\
\hline & \multirow{2}{*}{$\begin{array}{l}\text { Left arm } \\
\text { paresis }\end{array}$} & Score 1 & $14.1 \%$ & \multirow{2}{*}{$83 \%$} \\
\hline & & Score 2 & $24.3 \%$ & \\
\hline & \multirow{2}{*}{$\begin{array}{l}\text { Right leg } \\
\text { paresis }\end{array}$} & Score 1 & $17.3 \%$ & \multirow{2}{*}{$89.1 \%$} \\
\hline & & Score 2 & $17.8 \%$ & \\
\hline & \multirow{2}{*}{$\begin{array}{l}\text { Left leg } \\
\text { paresis }\end{array}$} & Score 1 & $16 \%$ & \multirow{2}{*}{$83.4 \%$} \\
\hline & & Score 2 & $21.8 \%$ & \\
\hline & \multirow{2}{*}{ Sensory } & Score 1 & $34.6 \%$ & \multirow{2}{*}{$81.5 \%$} \\
\hline & & Score 2 & $24.8 \%$ & \\
\hline & \multirow{2}{*}{ Speech } & Score 1 & $43.5 \%$ & \multirow{2}{*}{$67.2 \%$} \\
\hline & & Score 2 & $23.9 \%$ & \\
\hline
\end{tabular}

Table 2. The relationship between continuous variables and stroke

\begin{tabular}{|c|c|c|c|c|c|}
\hline \multirow{3}{*}{ Variable } & \multicolumn{4}{|l|}{ Stroke } & \multirow{3}{*}{$p$-value* } \\
\hline & \multicolumn{2}{|c|}{ Positive (n=618) } & \multicolumn{2}{|c|}{ Negative $(n=265)$} & \\
\hline & Mean & SD & Mean & SD & \\
\hline Age (years) & 68.22 & 13.29 & 64.85 & 14.24 & $<0.01$ \\
\hline Blood sugar level (mg/dL) & 152.41 & 67.95 & 165.28 & 96.41 & 0.04 \\
\hline $\begin{array}{l}\text { Duration from symptom onset } \\
\text { to arrival at ED (hour) }\end{array}$ & 4.21 & 6.71 & 11.92 & 10.09 & $<0.01$ \\
\hline
\end{tabular}

${ }^{*} p$-value refers to the relationship between each continuous variable and final diagnosis of stroke 
Table 3. Relationship between CPSS and PreHAST items and final diagnosis of patient

\begin{tabular}{|c|c|c|c|c|}
\hline \multicolumn{2}{|l|}{ Item } & \multicolumn{2}{|c|}{ Stroke } & \multirow[b]{2}{*}{ p-value* } \\
\hline & & \multirow{2}{*}{$\begin{array}{c}\begin{array}{c}\text { Positive } \\
\text { (n=618) }\end{array} \\
315\end{array}$} & \multirow{2}{*}{$\begin{array}{c}\begin{array}{c}\text { Negative } \\
(\mathrm{n}=265)\end{array} \\
225\end{array}$} & \\
\hline \multirow{2}{*}{ Facial droop } & Normal & & & \multirow{2}{*}{$<0.01$} \\
\hline & Abnormal & 303 & 40 & \\
\hline \multirow{2}{*}{ Speech } & Normal & 139 & 161 & \multirow{2}{*}{$<0.01$} \\
\hline & Abnormal & 479 & 104 & \\
\hline \multirow{2}{*}{ Arm drift } & Normal & 107 & 62 & \multirow{2}{*}{0.04} \\
\hline & Abnormal & 511 & 203 & \\
\hline \multirow{2}{*}{ Commands } & Both correct $(0)$ & 515 & 257 & \multirow{2}{*}{$<0.01$} \\
\hline & One or none correct (2) & 103 & 8 & \\
\hline \multirow{2}{*}{ Eye position } & Normal (0) & 518 & 264 & \multirow{2}{*}{$<0.01$} \\
\hline & The patient 'gaze' at one side without purpose (2) & 100 & 1 & \\
\hline \multirow{2}{*}{ Visual field } & Normal (0) & 585 & 260 & \multirow{2}{*}{0.01} \\
\hline & Perceives only waving on one side (2) & 33 & 5 & \\
\hline \multirow{2}{*}{ Facial palsy } & Normal (0) & 314 & 203 & \multirow{2}{*}{$<0.01$} \\
\hline & One corner of the mouth hanging (1) & 304 & 62 & \\
\hline \multirow{3}{*}{$\begin{array}{l}\text { Right arm } \\
\text { paresis }\end{array}$} & Holds for 10 seconds $(0)$ & 400 & 238 & \multirow{3}{*}{$<0.01$} \\
\hline & Drifts but does not reach bed in 10 seconds (1) & 109 & 25 & \\
\hline & Drifts and reaches bed in 10 seconds or falls immediately (2) & 109 & 2 & \\
\hline \multirow{3}{*}{$\begin{array}{l}\text { Left arm } \\
\text { paresis }\end{array}$} & Holds for 10 seconds $(0)$ & 381 & 220 & \multirow{3}{*}{$<0.01$} \\
\hline & Drifts but does not reach bed in 10 seconds (1) & 87 & 27 & \\
\hline & Drifts and reaches bed in 10 seconds or falls immediately (2) & 150 & 18 & \\
\hline \multirow{3}{*}{$\begin{array}{l}\text { Right leg } \\
\text { paresis }\end{array}$} & Holds for 5 seconds $(0)$ & 401 & 236 & \multirow{3}{*}{$<0.01$} \\
\hline & Drifts but does not reach bed in 5 seconds (1) & 107 & 25 & \\
\hline & Drifts and reaches bed in 5 seconds or falls immediately (2) & 110 & 4 & \\
\hline \multirow{3}{*}{$\begin{array}{l}\text { Left leg } \\
\text { paresis }\end{array}$} & Holds for 5 seconds (0) & 384 & 221 & \multirow{3}{*}{$<0.01$} \\
\hline & Drifts but does not reach bed in 5 seconds (1) & 99 & 26 & \\
\hline & Drifts and reaches bed in 5 seconds or falls immediately (2) & 135 & 18 & \\
\hline \multirow{3}{*}{ Sensory } & Normal (0) & 251 & 216 & \multirow{3}{*}{$<0.01$} \\
\hline & Perceives less or different on one side (1) & 214 & 45 & \\
\hline & Perceives only on one side (2) & 153 & 4 & \\
\hline \multirow{3}{*}{ Speech } & Normal (0) & 201 & 178 & \\
\hline & Slight or moderate dysarthria or aphasia. Communication possible (1) & 269 & 79 & $<0.01$ \\
\hline & Severe dysarthria or aphasia. Communication not intelligible (2) & 148 & 8 & \\
\hline
\end{tabular}

${ }^{*} \mathrm{p}$-value refers to the relationship between each item and the final diagnosis of stroke 
The best predictor of stroke in CPSS with a maximum sensitivity of $78.5 \%$ and specificity of $66 \%$ was a cut-off point of 1.5. In a cut-off point of 1.5 , the highest sensitivity was $78.5 \%$ and in a cut-off point of 2.5 , the highest sensitivity was $91.3 \%$. (AUC: $0.744, p<0.01$ ) (Table 5, Figure 1).

The best predictor score for stroke in PreHAST was a cut-off point of 2.5-5.5, and it has the highest sensitivity (68.4\%) in cut-off point of 2.5 and highest specificity $(90.2 \%)$ in a cut-off point of 5.5 (AUC: 0.775$)(p<0.01)$ (Table 5, Figure 2).

\section{Discussion}

In our study, CPSS and PreHAST had high sensitivity and specificity in diagnosing stroke. CPSS had the highest sensitivity at a cut-off point of 1.5 and the highest specificity at a cut-off point of 2.5. PreHAST had the best predictor status at cut-off points of 2.5, 3.5, 4.5 and 5.5. On both tests, with an increase in cut-off points, the sensitivity was decreased and the specificity was increased.

Andsberg et al investigated the ability of PreHAST in diagnosing stroke and transient ischaemic attack. They showed that the test required 2-3 minutes to execute and a positive PreHAST increased the diagnostic sensitivity and specificity up to $100 \%$ and $40 \%$, respectively. It also revealed that PPV was
$50 \%$ among patients with a positive test, and NPV was $100 \%$. The ROC analysis showed an AUC of 0.77 . The best predictor was for PreHAST with 5 points that had $90 \%$ specificity and $20 \%$ sensitivity. The study attributed high limitation of CPSS to its fewer items as it could not evaluate isolated hemianopia, neglect or leg paresis (16). The results were similar to the results in our study.

Zhao et al investigated pre-hospital measures such as CPSS in the diagnosis of stroke among 565 patients with suspicion of stroke. They showed that the sensitivity and specificity of CPSS in diagnosing stroke with minimum score of 2 was $56 \%$ and $86 \%$, respectively, with an AUC of 0.71 (28). Our study also showed the same results.

In another study conducted by Katz et al, the ability of CPSS in diagnosing stroke was assessed and showed that CPSS is a test with high sensitivity and favorable specificity. This test can evaluate the patient faster due to its dichotomous answers. They found that CPSS with a score of equal or more than 2 (AUC of 0.89 ) diagnosed moderate stroke (NIHSS $\geq 10$ ) with lower sensitivity $(75 \%)$ and higher specificity $(85 \%)$ in comparison with severe stroke (NIHSS 15) with a sensitivity of $89 \%$ and specificity of $73 \%$ (19). In contrast, in our study the specificity was higher in higher scores and sensitivity was higher in lower scores.

Table 5. Results from the ROC-curve analysis for various cut-off points of CPSS and PreHAST

\begin{tabular}{|c|c|c|c|c|c|c|c|c|}
\hline \multicolumn{9}{|c|}{ Area under the curve } \\
\hline \multirow{2}{*}{$\begin{array}{l}\text { Test result } \\
\text { variable(s) }\end{array}$} & \multirow{2}{*}{ Area } & \multirow{2}{*}{ Cut-off } & \multirow{2}{*}{ Sensitivity } & \multirow[t]{2}{*}{ Specificity } & \multirow{2}{*}{$\begin{array}{l}\text { Std. } \\
\text { error }\end{array}$} & \multirow{2}{*}{$\begin{array}{l}\text { Asymptotic } \\
\text { sig. }\end{array}$} & \multicolumn{2}{|c|}{$\begin{array}{c}\text { Asymptotic } 95 \% \text { confidence } \\
\text { interval }\end{array}$} \\
\hline & & & & & & & Lower bound & Upper bound \\
\hline \multirow{2}{*}{ CPSS } & \multirow{2}{*}{.744} & 1.50 & $78.5 \%$ & $66 \%$ & \multirow{2}{*}{.018} & \multirow{2}{*}{.000} & \multirow{2}{*}{.709} & \multirow{2}{*}{.779} \\
\hline & & 2.50 & $36.4 \%$ & $91.3 \%$ & & & & \\
\hline \multirow{4}{*}{ Pre HAST } & \multirow{4}{*}{.775} & 2.50 & $68.4 \%$ & $76.2 \%$ & \multirow{4}{*}{.016} & \multirow{4}{*}{.000} & \multirow{4}{*}{.745} & \multirow{4}{*}{.806} \\
\hline & & 3.50 & $63.8 \%$ & $81.9 \%$ & & & & \\
\hline & & 4.50 & $56.5 \%$ & $86.4 \%$ & & & & \\
\hline & & 5.50 & $47.6 \%$ & $90.2 \%$ & & & & \\
\hline
\end{tabular}

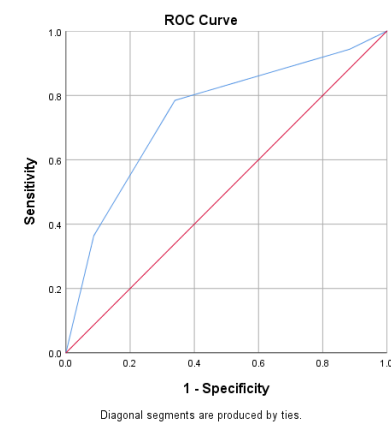

Figure 1. ROC curve of CPSS

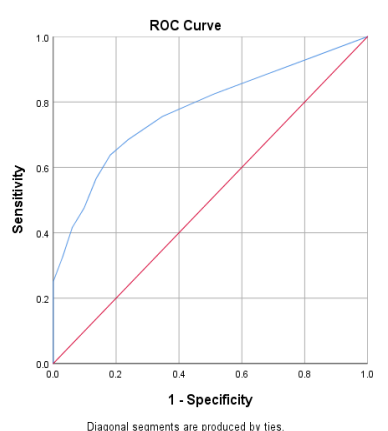

Figure 2. ROC curve of PreHAST 
Studnek et al investigated the validity of CPSS and Medic Prehospital Assessment for Code Stroke (Med PACS) in prehospital diagnosis of stroke among 461 suspected patients. They indicated that the sensitivity of CPSS was $79 \%$, higher than Med PACS with a sensitivity of $74 \%$. But the specificity of both tests was low: $23.9 \%$ and $32.6 \%$, respectively. In our study we found a more valuable role for CPSS in diagnosing stroke by differentiation of two effective cut-off points with higher sensitivity and specificity. The difference between the results of the two studies may be due to the lower study population in the study of Studnek (29).

In a study by Maddali et al the validity of CPSS was assessed in comparison to the findings on computed tomography (CT) among 66 patients with suspected stroke. They revealed that 62 patients (93\%) had stroke and the sensitivity of CPSS was $81 \%$ when combined with a CT scan. Only seven patients (11\%) with an abnormal CT scan had zero points in the CPSS test. The study showed that CPSS items including facial droop, arm drift and speech were impaired in $71 \%$ and $80 \%$ and $74 \%$ of patients, respectively. Sensitivity and specificity for facial droop was $75.8 \%$ and $100 \%$; arm drift $79.03 \%$ and $75 \%$, and for speech it was $74.1 \%$ and $100 \%$, respectively (30). Our study also showed that the highest sensitivity for arm drift and the highest specificity for facial droop.

Kummer et al investigated CPSS sensitivity and specificity among 751 patients with acute ischaemic stroke in the United States. They showed that using the cut-off point of 2, CPSS had $87.2 \%$ sensitivity and $94.3 \%$ specificity in diagnosing severe stroke (31). Accordingly, our study showed that the highest specificity was a cut-off point of 2.5. Removal of some patients before final diagnosis and high missing rate of some variables were problems encountered in this study. It is hoped that in future studies, sensitivity and specificity of various tests be differentiated for different types of stroke and true and false negative causes also be investigated.

\section{Conclusion}

Both PreHAST and CPSS are useful screening tools in the prehospital diagnosis of stroke. In addition to high sensitivity, these tests provide a grading system in which higher cut-off points lead to higher specificity.

\section{Competing interests}

The authors declare no competing interests. Each author of this paper has completed the ICMJE conflict of interest statement.

\section{References}

1. Peisker T, Koznar B, Stetkarova I, Widimsky P. Acute stroke therapy: a review. Trends Cardiovasc Med 2017;27:59-66.

2. Sheppard JP, Mellor RM, Greenfield S, et al. The association between prehospital care and in-hospital treatment decisions in acute stroke: a cohort study. Emerg Med J 2015;32:93-9.

3. Ebinger $\mathrm{M}$, Harmel $\mathrm{P}$, Nolte $\mathrm{CH}$, et al. Berlin prehospital or usual delivery of acute stroke care - study protocol. Int J Stroke 2017;12:653-8.

4. Thom T, Haase N, Rosamond W, et al. Heart disease and stroke statistics - 2006 update: a report from the American Heart Association Statistics Committee and Stroke Statistics Subcommittee. Circulation 2006;113:e85-151.

5. Baratloo A, Rahimpour L, Abushouk Al, et al. Effects of telestroke on thrombolysis times and outcomes: a metaanalysis. Prehosp Emerg Care 2018;22:472-84.

6. Baratloo A, Forouzanfar MM, Hashemi B, et al. Tissue plasminogen activator: a literature review. Arch Neurosci 2016;3:e30452.

7. Saver JL. Time is brain quantified. Stroke 2006;37:263-6.

8. Perez de la Ossa N, Ribo M, Jimenez X, Abilleira S. Prehospital scales to identify patients with large vessel occlusion: it is time for action. ibid. 2016;47:2877-8.

9. Fassbender K, Balucani C, Walter S, et al. Streamlining of prehospital stroke management: the golden hour. Lancet Neurol 2013;12:585-96.

10. Momeni M, Vahidi E, Seyedhosseini J, et al. Emergency overcrowding impact on the quality of care of patients presenting with acute stroke. Adv J Emerg Med 2018;2:e3.

11. Elfil M, Eldokmak M, Baratloo A, et al. Pathophysiologic mechanisms, neuroimaging and treatment in wake-up stroke. CNS Spectr 2019;1-8.

12.Powers WJ, Rabinstein AA, Ackerson T, et al. 2018 guidelines for the early management of patients with acute ischemic stroke: a guideline for healthcare professionals from the American Heart Association/American Stroke Association. Stroke 2018;49:e46-110.

13. Walter S, Kostopoulos P, Haass A, et al. Diagnosis and treatment of patients with stroke in a mobile stroke unit versus in hospital: a randomised controlled trial. Lancet Neurol 2012;11:397-404.

14. Garnett AR, Marsden DL, Parsons MW, et al. The rural Prehospital Acute Stroke Triage (PAST) trial protocol: a controlled trial for rapid facilitated transport of rural acute stroke patients to a regional stroke centre. Int J Stroke 2010;5:506-13.

15. Kasmaei HD, Baratloo A, Nasiri Z, Soleymani M, Yazdani MO. Recombinant tissue plasminogen activator administration in patients with cerebrovascular accident; a case series. Arch Neurosci 2015;2:e23315.

16. Andsberg G, Esbjornsson M, Olofsson A, et al. Prehospital ambulance stroke test - pilot study of a novel stroke test. Scand J Trauma Resusc Emerg Med 2017;25:37.

17. Rudd M, Buck D, Ford GA, Price Cl. A systematic review of stroke recognition instruments in hospital and prehospital settings. Emerg Med J 2016;33:818-22.

18. Togher FJ, Davy Z, Siriwardena AN. Patients' and ambulance service clinicians' experiences of prehospital care for acute myocardial infarction and stroke: a qualitative study. ibid. 2013;30:942-8. 


\section{References (continued)}

19. Katz BS, McMullan JT, Sucharew H, Adeoye O, Broderick JP. Design and validation of a prehospital scale to predict stroke severity: Cincinnati Prehospital Stroke Severity Scale. Stroke 2015;46:1508-12.

20. Itrat A, Taqui A, Cerejo $R$, et al. Telemedicine in prehospital stroke evaluation and thrombolysis: taking stroke treatment to the doorstep. JAMA Neurol 2016;73:162-8.

21.Paybast S, Ashraf A, Sarshad H, Shakiba M, Moadabi Y. Propagating relationship of cerebral oximetric volume and the clinical outcome of recombinant tissue plasminogen activator ( $r$-TPA) therapy on acute cerebral ischemic stroke Patients. Adv J Emerg Med 2020;4:e7.

22.Liberman AL, Prabhakaran S. Stroke chameleons and stroke mimics in the emergency department. Curr Neurol Neurosci Rep 2017;17:15.

23.Valenzuela Espinoza A, Van Hooff RJ, De Smedt A, et al. Development and pilot testing of 24/7 in-ambulance telemedicine for acute stroke: prehospital stroke study at the Universitair Ziekenhuis Brussel-Project. Cerebrovasc Dis 2016;42:15-22.

24.Purrucker JC, Hametner C, Engelbrecht A, et al. Comparison of stroke recognition and stroke severity scores for stroke detection in a single cohort. J Neurol Neurosurg
Psychiatry 2015;86:1021-8.

25.Bergs J, Sabbe M, Moons P. Prehospital stroke scales in a Belgian prehospital setting: a pilot study. Eur J Emerg Med 2010;17:2-6.

26.Zhelev Z, Walker G, Henschke N, Fridhandler J, Yip S. Prehospital stroke scales as screening tools for early identification of stroke and transient ischemic attack. Cochrane Database Syst Rev 2019;4:Cd011427.

27. Brandler ES, Sharma M, Sinert RH, Levine SR. Prehospital stroke scales in urban environments: a systematic review. Neurology 2014;82:2241-9.

28.Zhao H, Coote S, Pesavento L, et al. Large vessel occlusion scales increase delivery to endovascular centers without excessive harm from misclassifications. Stroke 2017;48:568-73.

29.Studnek JR, Asimos A, Dodds J, Swanson D. Assessing the validity of the Cincinnati prehospital stroke scale and the medic prehospital assessment for code stroke in an urban emergency medical services agency. Prehosp Emerg Care 2013;17:348-53.

30.Maddali A, Razack FA, Cattamanchi S, Ramakrishnan TV. Validation of the Cincinnati Prehospital Stroke Scale. J Emerg Trauma Shock 2018;11:111-4.

31.Kummer BR, Gialdini G, Sevush JL, et al. External validation of the Cincinnati Prehospital Stroke Severity Scale. J Stroke Cerebrovasc Dis 2016;25:1270-4. 